Review

\title{
Host Cell and Antibody Response towards Pasteurella Multocida B2 Infection-A General Review
}

\author{
${ }^{1}$ Eric Lim Teik Chung, ${ }^{1,2}$ Faez Firdaus Jesse Abdullah, \\ ${ }^{3,4}$ Yusuf Abba, ${ }^{1,5}$ Ali Dhiaa Marza, ${ }^{1,6}$ Hayder Hamzah Ibrahim, \\ ${ }^{3}$ Mohd Zamri-Saad, ${ }^{1,2}$ Abdul Wahid Haron, ${ }^{1}$ Abdul Aziz Saharee and ${ }^{3}$ Mohd Azmi Mohd Lila \\ ${ }^{I}$ Department of Veterinary Clinical Studies, Faculty of Veterinary Medicine, \\ ${ }^{2}$ Research Centre for Ruminant Disease, \\ ${ }^{3}$ Department of Veterinary Pathology and Microbiology, Faculty of Veterinary Medicine, \\ Universiti Putra Malaysia, 43400 Serdang, Selangor, Malaysia \\ ${ }^{4}$ Department of Veterinary Medicine, Faculty of Veterinary Medicine, \\ University of Maiduguri, PMB1069, Borno State, Nigeria, \\ ${ }^{5}$ Department of Veterinary Internal Medicine, \\ Faculty of Veterinary Medicine, Al-Qasim Green University, Iraq \\ ${ }^{6}$ Technical Institute Babil, Al Furat Alawast Technical University, Iraq
}

Article history

Received: 9-01-2015

Revised: 14-05-2015

Accepted: 18-06-2015

Corresponding Auhtor: Faez Firdaus Jesse Abdullah Department of Veterinary Clinical Studies, Faculty of Veterinary Medicine,

Universiti Putra Malaysia,

43400 Serdang, Selangor,

Malaysia

Tel: +60175550870

Email: jesseariasamy@gmail.com

\begin{abstract}
Pasteurella multocida was first shown to be the causative agent of fowl cholera by Louis Pasteur in 1881. Since then, this Gramnegative bacterium has been identified as the causative agent of many other economically important diseases in a wide range of hosts. Haemorrhagic Septicaemia (HS) is a disease, caused by Pasteurella multocida serotypes B2 and E2 that causes acute, highly fatal septicaemia with high morbidity and mortality in cattle and more susceptible in buffaloes. In this review, we aim to determine the host cell and antibody response towards Pasteurella multicoda B2 infection causing Haemorrhagic septicaemia.
\end{abstract}

Keywords: Haemorrhagic Septicaemia, Host Response, Antibody Response

\section{Introduction}

Haemorrhagic Septicaemia (HS) is an acute, fatal and septicaemic disease of cattle and buffaloes caused by specific serotype of Pasteurella multocida which is a Gram-negative coccobacillus (DeAlwis, 1999; OIE, 2008; 2009). Using a combination of capsular and somatic typing, the two common HS serotypes popularly known as the Asian and African serotypes are designated B2 and E2 respectively (Carter, 1962; Heddleston et al., 1972; Rimler et al., 1987). Buffaloes are more susceptible to the disease and usually occur more frequently in poor husbandry conditions and in countries with disease surveillance that is not well developed (DeAlwis, 1981; 1999; Farooq et al., 2011). Other stress factors causing outbreaks include exhaustion, chilling, malnutrition, dietary change, transportation, water deprivation, confinement, debilitating infections and overcrowding (Lane et al., 1992). The disease is of great economic importance in Malaysia and throughout Southeast Asia where cattle and buffaloes are abundant for beef and milk production (Thomas, 1972; Saharee et al., 1993; Kahn and Line, 2005). Besides that, economic losses due to HS also affect the rice production due to the high prevalence among draught animals (Benkirane et al., 2002). HS disease is a specific form of pasteurellosis in cattle and buffalo which is different from other pasteurelloses that play only a secondary role (DeAlwis, 1992).

In most cases, the clinical findings are either acute or peracute, resulting death within 8 to $24 \mathrm{~h}$ after onset (Kahn and Line, 2005; Annas et al., 2014). Sudden death is usually the first report of outbreaks among free ranging animals. There are four clinical syndromes in a disease animal. Animal will first found with elevated temperature above $40^{\circ} \mathrm{C}$, followed by submandibular edema and then respiratory distress with profuse nasal discharge and finally recumbency and death (DeAlwis, 1999). In an experiment done by DeAlwis (1992), buffaloes infected through aerosol and direct contact with diseased buffaloes showed clinical sign after 24 and $48 \mathrm{~h}$ respectively. Animals first show sign of dullness, then reluctant to move, fever, salivation and serous nasal 
discharge after infected with Pasteurella multocida B2. Edematous swelling is frequently seen, beginning in the throat region and spread to the parotid, neck and brisket region. Mucous membrane will then be congested and respiratory distress will occurs where animals will usually die within hours (DeAlwis, 1999; Lane et al., 1992). For post mortem lesions, the most obvious lesions in affected animals are the edema, widely distributed haemorrhages and general hyperaemia. In most cases, there will be also clear or straw coloured edematous fluid at the head, neck, brisket and musculature region. Petechial haemorrhages are particularly prominent in the pharyngeal and cervical lymph nodes. Besides that, blood tinge fluid is often found in the pericardial sac, thoracic and abdominal cavity (Kahn and Line, 2005; Abubakar et al., 2012). Meningitis was also observed by Lane (1992).

Clinical diagnosis of HS can be determined based on combinations of clinical signs, gross pathology lesions and laboratory diagnosis (DeAlwis, 1999). Pure cultures of Pasteurella multocida can be isolated and identify easily using streaking materials on to artificial media for morphological, cultural and biochemistry characteristic (OIE, 2008; 2009). Other diagnostic tests available include serological, biochemistry and molecular technique. For example, Polymerase Chain Reaction (PCR) was used as diagnostic tool to detect Pasteurella multocida $\mathrm{B} 2$ in mice infected with contaminated river water. Treatment of HS with antibiotic is of limited value unless it is done in the very early stage (DeAlwis, 1999; Kahn and Line, 2005). This is because all clinical cases are not detected in the early stage. Nonetheless, Pasteurella multocida organism is sensitive to variety of antimicrobial drugs such as sulfonomides, streptomycin and oxytetracycline. According to a case report by Lane (1992), early treatment of penicillin-streptomycin combination or one dose of long acting oxytetracycline given to affected animals were effective in controlling the clinical signs and mortality rate. As for prevention and control, vaccination is the most effective method in controlling the disease. The three common vaccine types used against HS are bacterins, Alum-Precipitated Vaccine (APV) and Oil-Adjuvanted Vaccine (OAV), (OIE, 2008). In Malaysia, OAV was used since 1966 as the main prophylactic agent and broth bacterin was only used mainly to provide immediate protection to susceptible animals during an outbreak (Thomas, 1972). OAV was proved to give up to $90 \%$ protection. This was confirmed by a study done by Bhatti (2005), where haemorrhagic septicaemia OAV was administered in Swiss albino mice via subcutaneously. The duration of immunity after administration of OAV is six to nine months and annual booster is required (OIE, 2008; 2009). Eradication of haemorrhagic septicaemia disease is not possible due to the existence of latent carriers (DeAlwis, 1999).

There are still many grey areas in our knowledge of haemorrhagic septicaemia, HS. According to DeAlwis (1999), significant gaps exist in the understanding of pathogenesis of the disease and some aspect of the epidemiology and immunology. A better understanding of these areas will lead to more effective control of the disease where it will assist the improvement of the vaccines and vaccination methods. In this review, we aim to determine the host cell and antibody response towards Pasteurella multicoda B2 infection causing HS by looking at the histopathology, haematology and biochemistry, acute phase proteins and antibody changes.

\section{Host Cell and Antibody Response}

\section{Histopathology}

Histology is a subject that focuses on the interrelationship and integration of molecular and physiological activities within the body (Samuelson, 2007; VanDijk et al., 2007). The earliest report on the histopathological changes in HS was done by Rhoades et al. (1967) who produced the disease experimentally in bison calf using B2 strain. The histopathological lesions such as haemorrhage, hyperaemia, oedema and white blood cells infiltration were observed in the lung, lymph nodes, spleen, gastro-intestinaltract, liver, kidney and the heart.

An experiment on clinico-pathlogy features in mice after oral inoculation with Pasteurella multocida B2 was done by Jesse et al. (2013a). The purpose of the study was to provide more knowledge and understanding on the pathogenicity as well as host response towards the Pasteurella mltocida in mice model. As for the findings, the lethality rate of Pasteurella multocida B2 group was $69.64 \%$ throughout 120 h. Histopathological findings revealed edema, inflammation, degeneration and necrosis were all observed in the lung, liver, heart, kidney, small intestine and colon of the treatment group. As stated earlier, HS is an acute, fatal and septicaemic disease that usually affects the respiratory route (DeAlwis, 1999). The high survival rate and lesions found in the gastrointestinal tract in this study were some interesting data. Jesse et al. (2013b) had conducted another study in cattle model. The aim of the study was to investigate the clinic-pathology in calves after infection with Pasteurella multocida and its immunogens Lipopolysaccharide (LPS) and Outer Membrane Protein (OMP) via intramuscular route. Oedema, inflammatory cells, degeneration, necrosis and haemorrhage of the lung, liver and heart were observed in all of the treatment groups. Therefore, it can be concluded that calves in the positive control groups showed evidence of host cell response after inoculation with Pasteurella multocida whole cells, LPS and OMP (Jesse et al., 2013b).

Another study on clinic-pathological changes in buffalo calves following oral and intra-thracheal 
exposure to Pasteurella multocida B2 was done by Abubakar et al. (2011) and Abubakar et al. (2012). All buffaloes inoculated orally survived throughout the seven days experiment. Histology observations showed mild to moderate congestion, oedema, neuthrophilic infiltration and present of bacterial colonies in the respiratory and gastrointestinal tract. In these two studies, the severity of histological lesions of orally infected group was milder compared to intra-tracheally infected group which is not a typical sign in HS infected animals. Oral route may not play a major role in the development of HS but they carried Pasteurella multocida organism in the gastrointestinal organs and may act as carrier animal (Abubakar et al., 2012).

In short, experiments done by a few researchers showed some interesting histological parameters included pulmonary oedema, presence of inflammatory cells, haemorrhage and necrosis in animals' organs after different route of inoculations in mice, cattle and buffaloes. The signs observed following oral exposure were much milder compared to intratracheal or respiratory routes (Abubakar et al., 2012). This can be a proof that oral route transmission can manipulate the Pasteurella multocida organism better compare to the other routes of inoculation. This indicates that the oral route could perhaps be a readily available route for effective vaccine administration and heightened the animals' immunity. However, knowledge on the tissue changes in the bone marrow and the immune system organs had yet to be uncovered. Information on this will play a significant role in getting better understanding and much knowledge on the pathogenesis of Pasteurella multocida organism.

\section{Haematology and Biochemistry}

Clinical pathology such as haematology and biochemistry is of great help to clinician in arriving at a correct diagnosis, prognosis and efficacy of a treatment (Sastry, 2006). During a bacterial infection, haematological and biochemistry changes are first detected during routine blood sampling. However, animal's defence mechanism can react quite differently and there is no singular pattern in complete blood count that indicates a bacterial infection (Weiss and Wardrop, 2010).

Jesse et al. (2013c) had reported that there were some haematological and biochemical markers that can be used for early detection in animals infected with wild type of Pasteurella multocida. Both studies were done in mice and cattle model respectively. In the mice model, there was significant increase in the band neutrophiles, eosinophils, plasma protein, thrombocytes, globulin, chloride and calcium level. However, the red blood cell counts, AP and albumin:globulin ration were found to be significantly decrease after infected with Pasteurella multocida organism via intra-peritoneal route. On the other hand, in the cattle model, different results were shown after intramuscular inoculation. Most of the parameters were found to be significantly decrease especially the white blood cells in the Pasteurella multocida treatment group. It is common for cattle with acute bacterial infections to have neutropenia because of the small storage pool of segmented neutrophils in the bone marrow (Jesse et al., 2013c).

In summary, whole cell of Pasteurella multocida and its immunogens LPS and OMP treatment groups showed changes in complete blood count and biochemisty profiles with various differences between groups. Haematology and biochemistry changes in the real host buffalo is still a query as previous experiments were only done only in mice and cattle model. More studies can be conducted to identify the haematology and biochemistry parameters during HS infection in buffaloes as these parameters could be used as HS indicators in the near future. With this, early detection can be done and treatment can be given effectively to prevent further losses to the farmers.

\section{Acute Phase Proteins}

Acute Phase Proteins (APPs) will be the first line of immune defence mechanism against preliminary infections of inflammations (Eckersall and Conner, 1988). However, the acute phase response measured in serum is nonspecific because it may be activated by a number of stimuli (Gronlund et al., 2003). These APPs will either increase or decrease in cases of infection and inflammation (Jesse et al., 2013b). This response occurs in all animals, but in different species the response of individual proteins can be significantly different (Eckersall and Conner, 1988). The APPs consist of "negative" and "positive" proteins that show a decrease or an increase in levels in response to challenge, respectively. The negative APPs include albumin, the most abundant constitutive of plasma protein and transferrin. The positive APPs include haptoglobin (Hp), C-reactive protein, Serum Amyloid A (SAA), caeruloplasmin, fibrinogen and alpha 1-acid glycoprotein (AGP). Hp is the major APPs in ruminants which show a negligible circulating level in normal animals, but increases over 100 fold on stimulation (Skinner et al., 1991). Hepatic production of APPs is also stimulated by pro-inflammatory cytokines for example interleukin 1, interleukin 6 and tumour necrosis factor alpha that are released into the circulation during acute phase response (Eckersall et al., 2008).

An experiment on APPs changes in mice was conducted by Jesse et al. (2013e). The purposed of the study was to evaluate the APPs profiles in mice associated with infection of Pasteurella multocida B2 and its immunogens via intra-peritoneal route. Mice of 
all treated groups showed significant changes in $\mathrm{Hp}$ along with SAA proteins; and showed no significant change in concentration of fibrinogen and C-reactive proteins. The whole cells only showed slight increase due to the short time of survival that did not provide enough time for inflammation to take place (Jesse et al., 2013e). Another mice model experiment was carry out to investigate the APPs responses in mice infected with river water contaminated by Pasteurella multocida type B2 via oral, aerosol and intra-peritoneal route. There was significant increase in the concentration of $\mathrm{Hp}$ and SAA level in mice inoculated intra-peritoneally. This was due to the increase in the production in APPs by the hepatocytes after infection.

Another experiment was done in cattle model by Jesse et al. (2013f) to evaluate the APPs response in relation to infection with Pasteurella multocida via intramuscular route. The positive and negative APPs were measured. There were significant increase in the $\mathrm{Hp}$ and SAA levels and significant decrease in the albumin level after intramuscular inoculation of Pasteurella multocida. The concentration of systemic APPs during inflammatory response appeared to be related to the severity of tissue damage and time course of the inflammatory process (Jesse et al., 2013f).

In short, intra-peritoneal route (Jesse et al., 2013e) and intramuscular (Jesse et al., 2013f) inoculation with Pasteurella multocida showed significant changes of APPs level in mice and cattle respectively. The oral route was less afflicted in causing disease in the mice model. The route of administration and stimulation of APPs changes in the real host buffalo is still unknown. Thus, more research could be done in the real host buffalo to determine the effective routes of administration for effective vaccine administration in the animal.

\section{Antibody}

Immunity is a process where the body protects against foreign organism or antigens (Studdert et al., 2011). The immune system is made of two important components which are the innate and humoral immunity. Innate immunity is also known as the first line of defence that prevents the entry of pathogens. Humoral immunity or the antibody-mediated immune system usually involves the production of specific antibodies against pathogen (Kumar, 2013). Morbidity and mortality due to HS in an endemic or epidemic area are largely dependent on the proportion of immune to nonimmune animals. Therefore, naturally acquired immunity plays a very important role in the different patterns of morbidity and mortality (DeAlwis, 1992). However, there is also relation between naturally acquired immunity and carrier status. Animals which develop high antibody titre after exposure to infection are the ones which become persistent carriers. In an experiment done by DeAlwis
(1992), some experimentally induced carriers animals did show mild transient signs of disease which were overcome. Thus, survivals from HS outbreaks are potential carriers that cause outbreaks from time to time.

A study measuring the IgG antibody in goats was done by Shafarin et al. (2008). The experiment was conducted to determine the proliferation and transmission pattern of Pasteurella multocida B2 via intranasal exposure and continuous dexamethasone injections. From the result, treatment groups that were injected with dexamethasone revealed significant increase in serum cortisol levels and slight decrease in the IgG levels. These findings were similar to previous study done by Zamri-saad et al. (1991) where dexamethasone was associated with stress in animals. Stress in animals will suppress the antibody production which will cause latent carrier to become an active carrier (DeAlwis, 1999). Another study was conducted by Zamri-saad et al. (2006), to determine the effect of intranasal exposure to low doses of Pasteurella multocida B2 stimulating antibody production against higher doses of the same organism. In this experiment, all goats showed low antibody response against Pasteurella multocida prior to intranasal exposure. After giving through intranasal route, treated group showed significant increase of antibody compared to the control group. Antibodies start to appear within approximately 5 to 7 days after exposure to an infectious agent. It takes nearly 2 weeks for a full antibody response to developed before the level start to decline (Zamri-saad et al., 2006).

Rafidah and Zamri-saad (2013), had conducted another experiment on the effect of dexamethasone on protection of vaccinated buffaloes against challenge by wild-type Pasteurella multocida B2. In this research, the IgG levels of vaccinated buffaloes were found to be increased significantly after intranasal vaccination using a live vaccine and remain high throughout the study period. On the other hand, dexamethasone injections did not significantly reduce the immune response in vaccinated animals but showed significant reduction in unvaccinated animals. Besides that, all vaccinated buffaloes injected with dexamethasone survived after they were challenged with wild type Pasteurella multocida. Thus, it was concluded that stress does not influence outbreaks of HS among vaccinated animals (Rafidah and Zamri-saad, 2013).

In summary, animals such as goat and buffalo exposed to Pasteurella multocida infection showed significant increase in the antibody IgG production (Shafarin et al., 2008; Zamri-saad et al., 2006). Administration of dexamethasone was found to cause slight decrease in the immune system but significant reduction in unvaccinated animals (Rafidah and Zamrisaad, 2013). Nevertheless, the antibody status of cattle 
and buffaloes during natural infection are still unknown as most studies were only done to measure vaccine efficacy. Besides that, mucosal antibody was also not determine in these few studies as Pasteurella multocida organisms have high efficacy in the respiratory tract where IgA plays a very important role during HS outbreak. IgA presents in the mucous membrane of the respiratory and gastro-intestinal tract (Susan et al., 1971; Ramsay et al., 1994; Macpherson et al., 2008; Kumar, 2013; Mestecky et al., 2014). Thus, could HS vaccine be administered through orally to stimulate a better antibody response in animal? If possible, oral route vaccination will allow farmers to cover higher vaccination percentage in buffaloes because the vaccine can be administered through feed. Zamri-saad (2013) had reported that only 17\% of Malaysia buffaloes were vaccinated due to the difficulty of vaccine administration. However, the development of oral vaccine will be able to control the outbreak of HS which cause major losses to farmers.

\section{Conclusion}

In conclusion, additional information on the aspect of histopathology, haematology and biochemistry, acute phase proteins and immunity on HS would be helpful in controlling the disease in the near future. Buffaloes are the most susceptible host for HS disease and by determine the host cell response promptly; prognosis of the animal will be better and treatment can be given more effectively.

\section{Funding Information}

The project was funded by Ministry of Higher Education, Malaysia (Grant no: 5524417).

\section{Author's Contributions}

FFJA, MZS, AWH, AAS, and MAML conceptualized and supervised this review paper. ELTC, YA, ADM and HHI collected data and drafted the manuscript. All authors have read and approved the manuscript.

\section{Ethics}

The authors declared no conflict of interest.

\section{References}

Abubakar, M.S. and M. Zamri-saad, 2011. Clinicopathological changes in buffalo calves following oral exposure to Pasteurella multocida B:2. Basic Applied Pathology, 2011: 130-135.

DOI: 10.1111/j.1755-9294.2011.01113.x

Abubakar, M.S., M. Zamri-saad and S. Jasni, 2012. Ultrastructural changes and bacterial localization in buffalo calves following oral exposure to Pasteurella multocida B: 2. Pakistan Vet. J., 33: 101-106.
Annas, S., M. Zamri-saad, F.F.A. Jesse and Z. Zunita, 2014. New sites of localisation of Pasteurella multocida B: 2 in buffalo surviving experimental haemorrhagic septicaemia. BMC Vet. Res., 10: 1-7. DOI: $10.1186 / 1746-6148-10-88$

Benkirane, A. and M.C.L. DeAlwis, 2002. Haemorrhagic septicaemia, its significance, prevention and control in Asia. Vet. Med. Czech, 2002: 234-240.

Bhatti, A.R., K. Tasneem, Z.J. Gill and A. Raza, 2005. Potency and safety testing of haemorrhagic septicaemia oil adjuvant vaccine in Swiss albino mice. Pakistan Vet. J., 25: 153-154.

Carter, G.R., 1962. Further observation on typing Pasteurella multocida by the indirect hemagglutination test. Can. J. Comp. Med. Vet. Sci., 26: 238-240.

DeAlwis, M.C.L., 1981. Mortality among cattle and buffaloes in Sri Lanka due to Haemorrhagic septicaemia, Trop. Anim. Hlth. Prod., 13: 195-202. DOI: $10.1007 /$ BF02237925

DeAlwis, M.C.L., 1992. Haemorrhagic septicaemia-A general review. Br. Vet. J., 148: 99-112. DOI: 10.1016/0007-1935(92)90101-6

DeAlwis, M.C.L., 1999. Haemorrhagic Septicaemia, Canberra, ACIAR.

Eckersall, P.D. and J.G. Conner, 1988. Bovine and canine acute phase protein. Vet. Res. Communi., 12: 169-178. DOI: 10.1007/BF00362798

Eckersall, P.D., F.P. Lawson, C.E. Kyle, M. Waterson and L. Bence et al., 2008. Maternal undernutrition and the ovine acute phase response to vaccination. BMC Vet. Res., 4: 1-10. DOI: 10.1186/1746-6148-4-1

Farooq, U., Z. Saeed, M.A. Khan, I. Ali and M.F. Qamar, 2011. Sero-surveillane of haemorrhagic septicaemia in buffaloes and cattle in Southern Punjab, Pakistan. Pak. Vet. J., 31: 254-256.

Gronlund, U., C. Hulten, P.D. Eckersall, C. Hogarth and K.P. Waller, 2003. Haptoglobin and serum amyloid $\mathrm{a}$ in milk and serum during acure and chronic experimentally induced Staphylococcus aures mastitis. J. Dairy Res., 70: 379-386. DOI: $10.1017 / \mathrm{S} 0022029903006484$

Heddleston, K.L., J.E. Gallagher and P.A. Rebers, 1972. Fowl Cholera: Gel diffusion precipitin test for serotyping Pasteurella multocida from avian species. Am. Association Avian Pathologists, 14: 925-936. DOI: 10.2307/1588773

Jesse, F.F.A., S.A. Affandi, A.Y. Osman, L. Adamu and M. Zamri-saad et al., 2013a. Clinico-pathological features in mice following oral exposure to Pasteurella multocida B: 2. IOSR J. Agric. Vet. Sci., 3: 35-39. 
Jesse, F.F.A., L. Adamu, A.Y. Osman, Z. Zakaria and R. Abdullah et al., 2013b. Clinico-pathological responses of calves associated with infection of Pasteurella multocida type B2 and the bacterial lipopolysaccharide and outer membrane protein immunogens. Int. J. Anim. Vet. Adv., 5: 190-198.

Jesse, F.F.A., A.Y. Osman, L. Adamu, Z. Zakaria and R. Abdullah et al., 2013c. Haematological and biochemical alterations in calves following infection with Pasteurella multocida type B2, bacterial lipopolysaccharide and outer membrane protein immunogens, Asian J. Anim. Vet. Adv., 8: 806-813. DOI: 10.3923/ajava.2013.806.813

Jesse, F.F.A., L. Adamu, A.Y. Osman, M. Zamri-saad and Z. Zakaria et al., 2013e. Acute phase protein profile and clinic-pathological changes in mice associated with the infection of Pasteurella multocida type $\mathrm{B}$ and the bacterial lipopolysaccharide and outer membrane protein imminogens. J. Anim. Vet. Adv., 12: 186-193.

Jesse, F.F.A., A.Y. Osman, L. Adamu, Z. Zakaria and R. Abdullah et al., 2013f. Acute phase protein Profiles in calves following infection with whole cell, lipopolysaccharide and outer membrane protein extracted from Pasteurella multocida type B: 2. Asian J. Anim. Vet. Adv., 8: 655-662. DOI: 10.3923 /ajava.2013.655.662

Kahn, C.M. and S. Line, 2005. The Merck Veterinary Manual, 9th Edn., USA, Merial.

Kumar, A., 2013. Textbook of Immunology, New Delhi, TERI.

Lane, E.P., N.D. Kock, F.W.G. Hill and K. Mohan, 1992. An outbreak of haemorrhagic septicaemia in cattle in Zimbabwe. Trop. Anim. Hlth. Prod., 24: 97-102. DOI: 10.1007/BF02356952

Macpherson, A.J., K.D. McCoy, F.E. Johansen and P. Brandtzaeg., 2008. The immune geography of $\operatorname{IgA}$ induction and function. Mucosal Immunity, 1: 11-22. DOI: $10.1038 / \mathrm{mi} .2007 .6$

Mestecky, J. and C.O. Elson, 2014. Peyer's patches as the inductive site for IgA responses, J. Immunology, 180:1293-1294. DOI: 10.4049/jimmunol.180.3.1293

OIE, 2008. Haemorrhagic Septicaemia. In Chapter 2: OIE Terrestrial Manual, pp: 739-751.

OIE, 2009. Haemorrhagic Septicaemia. Chapter 2.4.12: OIE Terrestrial Manual, pp: 739-750.

Rafidah, O. and M. Zamri-saad, 2013. Effect of dexamethasone on protective efficacy of live gdhA derivative Pasteurella multocida B: 2 vaccine. Asian J. Anim. Vet. Adv., 8: 548-554. DOI: 10.3923/ajava.2013.548.554

Ramsay, A.J., A.J. Husband, I.A. Ramshaw, S. Bao and K.I. Matthaei et al., 1994. The role of interleukin-6 in mucosal IgA antibody responses in vivo. Science, 264: 561-563. DOI: 10.1126/science. 8160012
Rhoades, K.R., K.L. Heddleston and P.A. Rebers, 1967. Experimental haemorrhagic septicaemia: Gross and microscopic lesions resulting from acute infections and from endotoxin administration. Canadian J. Comparative Med., 31: 226-233.

Rimler, R.B. and K.R. Rhoades, 1987. Serogroup F, a new capsule serogroup of Pasteurella multocida, J. Clinical Microbiol., 25: 615-618.

Saharee, A.A., N.B. Salim, A. Rasedee and M.R. Jainudeen, 1993. Haemorrhagic septicaemia carriers among cattle and buffalo in Malaysia. Pasteurellosis Production Animals, ACIAR Proceeding.

Samuelson, D.A., 2007. Textbook of Veterinary Histology, Missouri, Saunders Elsevier.

Sastry, G.A., 2006. Veterinary Clinical Pathology, New Delhi, CBS Publishers \& Distributors.

Shafarin, M.S., M. Zamri-saad, Siti-Khairani and A.A. Saharee, 2008. Proliferation and transmission patterns of Pasteurella multocida B2 in goats. Trop. Anim. Health Prod., 40: 335-340. DOI: $10.1007 / \mathrm{s} 11250-007-9111-4$

Skinner, J.G., R.A. Brown and Roberts, 1991. Bovine haptoglobin responses in clinically defined field conditions. J. Br. Vet. Assoc., 128: 147-149. DOI: $10.1136 /$ vr.128.7.147

Studdert, V.P., C.C. Gay and D.C. Blood, 2011. Saunders Comprehensive Veterinary Dictionary, 4th Edn., Elsevier Health Sciences, London, Saunders, ISBN-10: 0702047449, pp: 1344.

Susan, W.C. and J.J. Cebra, 1971. Payer's pathes: An enriched source of precursors for IgA producing immunocytes in the rabbit. J. Experim. Med., 134: 188-200. DOI: $10.1084 /$ jem.134.1.188

Thomas, J., 1972. The control of haemorrhagic septicaemia in west Malaysia. Trop. Anim. Hlth. Prod., 4: 95-101. DOI: 10.1007/BF02359741

VanDijk, J.E., E. Gruys and J.M.V.M. Mouwen, 2007. Color Atlas of Veterinary Pathology, 2nd Edn, Philadelphia, Saunders Elsevier.

Weiss, D.J. and K.J. Wardrop, 2010. Schalm's Veterinary Hematology. 6th Edn, Iowa, Wiley-Blackwell

Zamri-saad, M., Ernie, Z.A. and Sabri, M.Y., 2006. Protective effect following intranasal exposure of goats to live Pasteurella multocida B2. Trop Anim Health Prod., 38: 541-546. DOI: $10.1007 / \mathrm{s} 11250-006-4437-\mathrm{x}$

Zamri-saad, M., Jasni, S., Nurida, A.B. and A.R. SheikhOmar, 1991. Experimental infection of dexamethasone-treated goats with Pasteurella haemolytica A2. Br. Vet. J., 147: 565-568. DOI: 10.1016/0007-1935(91)90027-K

Zamri-saad, M., 2013. Haemorrhagic septicaemia of cattle and buffaloes in Asia. Universiti Putra Malaysia Press: Selangor. 